\title{
Correlação entre o Diagnóstico Morfologico Pré e Pós-natal das Nefrouropatias Fetais
}

Correlation between Pre- and Postnatal Morphologic Diagnosis of Fetal Nephrouropathies

Alamanda Kfoury Pereira ${ }^{1}$, Eduardo Araújo de Oliveira ${ }^{2}$, Henrique Vitor Leite ${ }^{1}$, Antônio Carlos Vieira Cabral ${ }^{1}$

\section{RESUMO}

Objetivo: avaliar a acuidade da ecografia antenatal no diagnóstico das nefrouropatias fetais. Pacientes e Métodos: os autores acompanharam prospectivamente 127 gestações, referidas ao Centro de Medicina Fetal da UFMG devido à suspeita de anomalia do trato urinário fetal. Procedeu-se então ao ultra-som morfológico, no qual procurou-se verificar a biometria, padrão de crescimento fetal, vitalidade, malformações associadas e, finalmente, uma descrição sistematizada do sistema renal fetal, visando-se estabelecer o diagnóstico pré-natal. Este foi posteriormente correlacionado com o diagnóstico obtido após o nascimento.

Resultados: utilizando-se o indice kappa (que mede a concordância entre diferentes medidas, métodos ou instrumentos de medição com os seguintes critérios: abaixo de 0,40, baixa concordância; entre 0,40 e 0,75, concordância razoável e acima de 0,75, excelente concordância), os autores encontraram excelente indice de concordância global entre o diagnóstico pré e pós-natal (indice kappa 95\%). Dos 127 casos, houve apenas 9 casos discordantes, todos referentes às uropatias obstrutivas, nos quais o diagnóstico pós-natal mostrou diferente nivel de obstrução (6 casos) ou confusão com rim multicístico (3 casos).

Conclusão: o estudo ecográfico sistematizado do sistema renal é um método eficaz de diagnóstico pré-natal das nefrouropatias, permitindo adoção de medidas que visem melhorar a evolução destes neonatos, desde o encaminhamento para centros especializados, antecipação do parto (nos casos em agravamento) até a terapia intra-útero (para preservar a função renal até que seja possivel o nascimento). O estudo ecográfico seriado e a amnioinfusão podem ser úteis para melhorar a precisão do diagnóstico pré-natal.

PALAVRAS-CHAVE: Malformações fetais. Ultra-sonografia. Diagnóstico pré-natal. Trato urinário fetal.

\section{Introdução}

Nas últimas décadas, o desenvolvimento de equipamentos ultra-sonográficos tem possibilitado o estudo minucioso de detalhes anatômicos do feto, permitindo um verdadeiro "exame fisico” pré-natal. A Organização Mundial de Saúde já reconhece o diagnóstico intra-útero como um procedimento do pré-natal ${ }^{1}$.

Verifica-se hoje conhecimento progressivo

$\overline{1,2}$ Trabalho realizado no Centro de Medicina Fetal, Departamento de Ginecologia e Obstetrícia da Faculdade de Medicina da UFMG Correspondência:

Alamanda Kfoury Pereira

Rua Quintiliano Silva, 253/ 401- Santo Antônio

30350-040 - Belo Horizonte - MG do embrião, do feto e de seu ambiente, sua morfologia, suas funções, seu bem-estar, além das possibilidades de diagnóstico biológico mediante obtenção de amostras de tecidos fetais. Desta forma, o feto passa a ser individualizado como um paciente, no qual as possibilidades de diagnóstico e tratamento intra-uterino efetivamente contribuem para a sua evolução perinatal.

Certamente, são as nefrouropatias o grupo de doenças que mais têm se beneficiado dos avanços técnicos da exploração ultra-sonográfica pré-natal ${ }^{2}$. O estudo ecográfico do sistema urinário fetal é bastante favorecido, seja devido ao contraste oferecido entre parte sólida e liquida de seus tecidos, seja pela natureza cística da maioria das lesões. 
Diferentemente de outras anomalias, as doenças renais dificilmente estão associadas a fatores extrinsecos, tais como agentes teratogênicos ou infecciosos, sendo na maioria das vezes de origem multifatorial. Algumas delas, entretanto, apresentam padrão de herança familiar e o risco de recorrência varia com a doença em questão, porém em sua maioria são esporádicos. Associam-se a anomalias cromossômicas em $2,5 \%$ dos casos, podendo chegar a $23 \%$ quando estão presentes outras anomalias ou o crescimento intra-uterino retardado ${ }^{3}$. Atualmente, estima-se em cerca de um para 200 a um para 1.000 nascimentos e de 0,3 a $17 \%$ das malformações diagnosticadas intra-útero ${ }^{4}$.

Trata-se de grupo importante e heterogêneo entre as doenças que acometem o concepto e cujo diagnóstico precoce se reveste de especial relevância. Primeiro, porque essas anomalias são, quase sempre, assintomáticas ao nascimento. Nos estudos de recém-nascidos sabidamente portadores de malformações do trato urinário (pela ultra-sonografia pré-natal), em apenas 10 a $30 \%$ dos casos os achados clínicos permitiriam a suspeita diagnóstica ${ }^{5}$. A doença freqüentemente é reconhecida tardiamente na infância, na vigência de complicações tais como infecção do trato urinário, hipertensão, atraso no crescimento e, algumas vezes, uma silenciosa e progressiva deterioração da função renal. As uropatias são uma das causas mais freqüentes de insuficiência renal na infância e adolescência, em todo o mundo, além de serem as únicas juntamente com as glomerulonefrites cujo tratamento precoce é capaz de prevenir ou adiar a perda crônica da função renal. Estudos no Brasil mostraram que cerca de $30 \%$ das crianças portadoras de insuficiência renal crônica apresentam alguma uropatia. Torna-se, portanto, evidente a necessidade de um diagnóstico precoce das uropatias ${ }^{6-8}$.

Finalmente, o diagnóstico pré-natal das uropatias traz a oportunidade de influenciar favoravelmente a evolução da gestação e da abordagem pré-natal, possibilitando desde a mudança do local do parto, a integração com nefrologistas pediátricos e equipe especializada, até o tratamento intra-útero, na tentativa de prevenir ou minimizar danos ao rim acometido ${ }^{9,10}$.

A descrição ecográfica do trato urinário teve início no início da década de 60 , juntamente com os primeiros relatos sobre a ultra-sonografia obstétrica ${ }^{11}$, tornando-se cada vez mais freqüentes as publicações de casos isolados e até séries das mais variadas anomalias do trato urinário fetal. É sabido hoje que o sucesso na abordagem perinatal deste grupo de doenças passa diretamente pelo diagnóstico morfológico correto da lesão e pela avaliação prognóstica da função renal.

Os estudos que avaliam a eficácia do diagnóstico pré-natal das nefrouropatias apresentam resultados bastante conflitantes. A dificuldade em compará-los deve-se à variada casuística, metodologia (retrospectivos ou prospectivos, equipamentos utilizados, experiência técnica dos examinadores) e principalmente às diferentes nomenclaturas utilizadas na definição do diagnóstico e dos achados ecográficos que caracterizam as diferentes doenças. Por exemplo, não há padronização na definição da hidronefrose (a maioria dos autores considera o diâmetro ânteroposterior da pelve renal acima de $10 \mathrm{~mm}$ ) ou quanto aos quadros obstrutivos, nos quais alguns estudos definem ecograficamente o refluxo e a obstrução propriamente dita, o que é muito dificil em termos de diagnóstico morfológico prénatal.

Assim, o presente estudo visa avaliar a acuidade da ecografia pré-natal no diagnóstico das anomalias do trato urinário fetal, por meio da descrição sistematizada dos seus componentes e da definição do tipo e natureza das suas lesões.

\section{Pacientes e Métodos}

Trata-se de estudo prospectivo, durante o qual, no período de janeiro de 1992 a janeiro de 1998, foram acompanhadas 127 gestantes, referenciadas de postos de atendimento pré-natal ao Centro de Medicina Fetal da UFMG devido à suspeita ecográfica de anomalia do trato urinário.

Estas pacientes foram então submetidas a exame ecográfico em aparelho Aloka SSP 650 CL. Foi realizado estudo sistemático do trato urinário, que incluiu o estudo morfológico dos rins (tamanho, localização, relações anatômicas, volume, aspecto do parênquima, ecogenicidade e estrutura dos cálices e bacinetes). Verificou-se a presença de dilatação da pelve renal, pela medida do diâmetro ântero-posterior, considerando-se hidronefrose quando este diâmetro fosse maior que $10 \mathrm{~mm}$. O estudo do trato urinário era completado com a visualização de dilatação do ureter e uretra e dilatação e/ou espessamento da parede vesical.

Os exames foram realizados em intervalos variáveis de acordo com a gravidade da lesão ou com a necessidade de se definir, baseado nos achados obtidos, o diagnóstico morfológico da nefrouropatia. Entre as doenças renais, encon- 
tramos: agenesia renal (uni ou bilateral), rim multicístico e rim policístico do tipo adulto e infantil. As doenças obstrutivas foram classificadas quanto ao nivel anatômico da lesão, não sendo possivel nem necessário no pré-natal definir- se a sua natureza (funcional ou estrutural). Sintetizamos na Tabela 1 as anomalias nefrourológicas encontradas, com os respectivos achados ecográficos que as definiram.

Tabela 1 - Critérios de diagnóstico morfológico das uropatias fetais.

\begin{tabular}{|c|c|c|c|c|c|}
\hline Diagnóstico & $\operatorname{Rim}$ & Ureter & Bexiga & Uretra & Líquido amniótico \\
\hline Agenesia renal unilateral (ARU) & Ausente (unilateral) & Normal & Normal & Normal & Normal \\
\hline Agenesia renal bilateral (ARB) & Ausente & Ausente & Ausente & Ausente & Reduzido \\
\hline Rim multicístico (RMC) & $\begin{array}{l}\text { Aumentado; macrocistos; } \\
\text { parênquima não-visualizado }\end{array}$ & Normal & Normal & Normal & Normal/reduzido \\
\hline Rim policístico adulto (RPC-a) & $\begin{array}{l}\text { Irregular; cistos variáveis; } \\
\text { parênquima hiperecogênico }\end{array}$ & Normal & Normal & Normal & Normal/ reduzido \\
\hline Rim policístico infantil (RPC-i) & Aumentado, com microcistos bileterais & Normal & Normal & Normal & Normal/ reduzido \\
\hline $\begin{array}{l}\text { Obstrução da junção uretero- } \\
\text { pélvica (OJUP) }\end{array}$ & Hidronefrose & Normal & Normal & Normal & Normal \\
\hline $\begin{array}{l}\text { Obstrução da junção uretero- } \\
\text { vesical (OJUV) }\end{array}$ & Hidronefrose & Dilatado & Normal & Normal & Normal \\
\hline Obstrução uretral (OU) & Normal ou hidronefrose & $\begin{array}{c}\text { Dilatado } \\
\text { bilateralmente }\end{array}$ & Megabexiga & $\begin{array}{l}\text { Dilatada } \\
\text { ou normal }\end{array}$ & Normal/ reduzido \\
\hline
\end{tabular}

O último exame realizado antes do parto foi então confrontado com os achados obtidos por estudo ecográfico, radiológico, cintilográfico ou por estudo anatomopatológico no caso de natimortos ou óbitos neonatais. Para verificação de concordância entre o diagnóstico pré e pósnatal foi utilizado o indice Kappa, que fornece a seguinte estimativa: abaixo de 0,40 , reflete baixa concordância; entre 0,40 e 0,75 , boa concordância, e acima de 0,75 , concordância excelente.

\section{Resultados}

Nas Tabelas 2 e 3 apresentamos a distribuição dos casos de acordo com o diagnóstico morfológico pré e pós-natal, respectivamente. Verifica-se que as anomalias obstrutivas são as mais prevalentes no pré-natal e que em conjunto correspondem a mais de $70 \%$ dos casos, seguidas da agenesia renal bilateral com 14 casos $(11,2 \%)$. Das doenças císticas do parênquima renal observa-se número significativo de casos de rim multicístico, com 12 dos 127 casos $(9,4 \%)$.

Pode-se constatar, na Tabela 3 , que as anomalias obstrutivas também estão entre as mais encontradas após o nascimento e que, em conjunto, totalizaram $85 / 127$ casos $(66,9 \%)$, seguindo-se o rim multicístico e a agenesia renal bilateral.

A Tabela 4 apresenta a correlação entre o diagnóstico morfológico pré e pós-natal. Pode-se verificar nas células sombreadas os casos em que houve concordância entre o diagnóstico prénatal e o obtido após o nascimento, considerado definitivo.

Tabela 2 - Distribuição dos casos conforme o diagnóstico morfológico pré-natal das uropatias.

\begin{tabular}{lrr}
\hline Diagnóstico morfológico pré-natal & $\mathbf{n}$ & $\%$ \\
\hline ARU & 1 & 0.8 \\
ARB & 14 & 11.2 \\
RMC & 12 & 9.4 \\
RPC-infantil & 7 & 5.6 \\
RPC-adulto & 1 & 0.7 \\
OJUP & 34 & 26.7 \\
OJUV & 28 & 22.0 \\
OU & 30 & 23.6 \\
Total & 127 & 100.0 \\
\hline
\end{tabular}

ARU-agenesia renal unilateral; ARB-agenesia renal bilateral; RMC-rim multicistico; RPC-rim policístico; OJUP-obstrução dajunção uretero-pélvica; OJUV-obstrução da junção ureterovesical; OU-obstrução uretral. 
Tabela 3 - Distribuição dos casos conforme o diagnóstico morfológico pósnatal das uropatias.

\begin{tabular}{lrr}
\hline Diagnóstico morfológico pós-natal & $\mathbf{n}$ & $\%$ \\
\hline ARU & 1 & 0,8 \\
ARB & 14 & 11,2 \\
RMC & 15 & 11,8 \\
RPC-infantil & 8 & 6,3 \\
RPC-adulto & 1 & 0,8 \\
OJUP & 30 & 23,6 \\
OJUV & 25 & 19,7 \\
OU & 30 & 23,6 \\
Outro & 1 & 0,8 \\
NAD & 2 & 1,6 \\
Total & 127 & 100,0 \\
\hline R & 27
\end{tabular}

ARU-agenesia renal unilateral; ARB-agenesia renal bilateral; RMC-rim multicístico; RPC-rim policístico; OJUP-obstrução da junção uretero-pélvica; OJUV-obstrução da junção ureterovesical; OU-obstrução uretral; NAD-nenhuma anomalia detectada.

Visando o estudo da concordância diagnóstica global das uropatias fetais, aplicou-se o método de kappa não-ponderado. $\mathrm{O}$ indice verifica- do foi de 0,95 , que representa excelente concordância entre o diagnóstico pré e pós-natal das anomalias do trato urinário.

Uma comparação de cada diagnóstico revela que o acerto foi de $100 \%$ nas seguintes doenças: agenesia renal bilateral, agenesia renal unilateral, rim policístico adulto e obstrução uretral. Nas demais doenças a concordância não foi absoluta.

Pode-se observar, ainda na Tabela 4, que nas doenças obstrutivas é que se concentram os equívocos no diagnóstico pré-natal. Dos 34 casos de obstrução da junção uretero-pélvica (OJUP), houve concordância em 29, o que corresponde a $85,3 \%$. Os cinco casos erroneamente diagnosticados como OJUP foram assim distribuídos: um caso de obstrução da junção uretero-vesical (OJUV), um de rim multicístico (RMC), um de rim policístico (RPC-infantil), um classificado como "outro", cujo diagnóstico pósnatal foi cisto renal simples, e, finalmente, um caso em que não foi vista qualquer anomalia na avaliação neonatal.

\begin{tabular}{|c|c|c|c|c|c|c|c|c|c|c|c|}
\hline Pós-natal Pré-natal & ARU & ARB & RMC & RPC-i & RPC-a & OJUP & OJUV & OU & Outro & NAD & Total \\
\hline ARU & 1 & & & & & & & & & & 1 \\
\hline ARB & & 14 & & & & & & & & & 14 \\
\hline RMC & & & 12 & & & & & & & & 12 \\
\hline RPC-i & & & & 7 & & & & & & & 7 \\
\hline RPC-a & & & & & 1 & & & & & & 7 \\
\hline OJUP & & & 1 & 1 & & 29 & 1 & & 1 & 1 & 34 \\
\hline OJUV & & & 2 & & & 1 & 24 & & & 1 & 28 \\
\hline OU & & & & & & & & 30 & & & 30 \\
\hline Total & 1 & 14 & 15 & 8 & & 30 & 25 & 30 & 1 & 2 & 127 \\
\hline
\end{tabular}

ARU-agenesia renal unilateral; $A R B$-agenesia renal bilateral; RMC-rim multicistico;

RPC-rim policístico; OJUP-obstrução da junção uretero-pélvica; OJUV-obstrução da junção uretero-vesical; OU-obstrução uretral; NAD-nenhuma anomalia detectada.

Entre os casos de OJUV, do total de 28 casos com diagnóstico pré-natal, ocorreram quatro erros de diagnóstico, correspondendo a uma concordância de 85,7\%. O diagnóstico pós-natal consistiu de um caso de OJUP, dois de RMC e um caso em que não foi vista anomalia à avaliação neonatal. Os casos discordantes podem ser vistos na Tabela 5 .

\section{Discussão}

As nefrouropatias estão entre as doenças mais prevalentes no período antenatal. Antes do avanço do diagnóstico pré-natal a sua incidência era subestimada, pois as estatísticas baseavam-se em dados pós-natais, não incluindo os óbitos intra-uterinos ou natimortos sem o devido esclarecimento diagnóstico. Atualmente, estima-se em cerca de um para 200 a um para 1000 nascimentos correspondendo a 0,3 a $17 \%$ das malformações diagnosticadas intra-útero ${ }^{4,11}$. O diagnóstico pré-natal é da maior importância, pois definem-se precocemente a natureza e características da doença, sua história natural e prognóstico, permitindo tomada de decisões que vão da conduta expectante nos casos incompativeis com a vida ou que se constata perda irreversível da função renal (doenças císticas re- 
nais bilaterais, agenesia renal ou obstruções com perda da função renal), passa pela transferência para centro terciário com antecipação do parto para tratamento pós-natal, nos casos (após 34 semanas) com obstrução do trato urinário fetal com função renal preservada, nas gestações próximas do termo, até a terapia fetal nos casos de obstrução do trato urinário em gestações longe do termo, com o objetivo de preservar a função renal fetal até a sua viabilidade.

Tabela 5 - Casos de Uropatias em que Houve Discordância Entre o Diagnóstico Prée ePós-natal.

\begin{tabular}{cccc}
\hline $\begin{array}{c}\text { Caso } \\
\mathbf{n}^{\mathbf{0}}\end{array}$ & $\begin{array}{c}\text { Idade gestacional } \\
\text { (semanas) }\end{array}$ & $\begin{array}{c}\text { Diagnóstico } \\
\text { pré-natal }\end{array}$ & $\begin{array}{c}\text { Diagnóstico } \\
\text { pós-natal }\end{array}$ \\
\hline 1 & 27 & OJUP & OJUV \\
2 & 29 & OJUP & RPC \\
3 & 31 & OJUP & Cisto Renal \\
4 & 30 & OJUP & Reversão \\
5 & 28 & OJUP & RMC \\
6 & 30 & OJUV & RMC \\
7 & 34 & OJUV & Reversão \\
8 & 29 & OJUV & OJUP \\
9 & 32 & OJUV & RMC \\
\hline
\end{tabular}

RMC-rim multicístico; RPC-rim policístico; OJUP-obstrução da junção uretero-pélvica; OJUV-obstrução da junção uretero-vesical.

Dessa maneira, a precisão do diagnóstico morfológico pré-natal é de fundamental importância, pois influenciará decisivamente na conduta e evolução perinatal daquele feto comprometido. Os estudos que fazem esta correlação pré e pós-natal divergem significativamente em vários aspectos, desde os critérios de seleção dos casos e a metodologia empregada até os critérios de diagnóstico e de nomenclatura, fato que limita uma análise comparativa.

$\mathrm{Na}$ Tabela 6 estão resumidos os resultados encontrados por vários autores, comparando com os do nosso estudo. Há uma grande variabilidade encontrada nestes estudos; a casuística (de 18 a 166 casos), o desenho metodológico, sendo alguns retrospectivos ${ }^{12-15}$, outros prospectivos ${ }^{16-20}$. Alguns estudos definem a hidronefrose pelo diâmetro ântero-posterior da pelve renal entre 5 e $10 \mathrm{~mm}$ como pielectasia, exigindo acompanhamento ${ }^{15}$, porém a maioria considera como sendo hidronefrose quando acima de $10 \mathrm{~mm}$. A distinção entre refluxo e obstrução também resultou em equívocos ao comparar com o diagnóstico pós-natal, uma vez que esta é bastante difícil no exame ecográfico prénatal $^{13,14}$. Preferimos padronizar os achados de dilatação do sistema coletor com o nome genéri- co de "obstrução" e definir o seu nível anatômico, já que na abordagem pré-natal interessa mais o nivel e progressividade da lesão e menos a natureza anatômica ou funcional da mesma.

Tabela 6 - Concordância Entre o Diagnóstico Pré e Pós-natal das Uropatias Fetais.

\begin{tabular}{lccccc}
\hline Ano & $\mathbf{n}$ & $\begin{array}{c}\text { Concordância } \\
(\%)\end{array}$ & $\begin{array}{c}\text { Tratamento } \\
\text { antenatal }\end{array}$ & $\begin{array}{c}\text { Sobrevida } \\
(\mathbf{\%})\end{array}$ & Referência \\
\hline 1983 & 44 & 96 & Sim & 50 & 16 \\
1988 & 18 & 66 & Sim & 50 & 17 \\
1989 & 48 & 91 & - & - & 18 \\
1989 & 92 & 50 & - & - & 19 \\
1990 & 27 & 84 & - & - & 20 \\
1991 & 73 & 35 & - & - & 12 \\
1993 & 46 & 78 & - & - & 21 \\
1994 & 125 & 60 & - & - & 13 \\
1995 & 166 & 100 & - & - & 14 \\
1996 & 142 & 66 & - & - & 15 \\
1998 & 127 & 95 & Sim $^{22}$ & $40 \%$ & Este estudo \\
\hline
\end{tabular}

Nosso estudo mostrou, dentro desta sistemática, indice de concordância global de 95\% entre o diagnóstico pré e pós-natal das nefrouropatias fetais. A despeito das variações metodológicas, observa-se boa concordância diagnóstica entre os estudos. O resultado favorável aqui encontrado deve-se a um somatório de fatores, entre eles o equipamento utilizado, o treinamento da equipe de ecografistas, que vem desde o início do estudo trabalhando de forma integrada, e a objetividade na definição dos critérios de diagnóstico.

Todos os casos desta série requereram mais de um exame ecográfico para estabelecer o diagnóstico. Em algumas patologias, como na agenesia renal bilateral e rim policístico tipo infantil, o diagnóstico pré-natal é de suma importância. Um falso-positivo ou negativo pode determinar condutas obstétricas antagônicas relativas à viabilidade fetal.

Fazendo uma análise por doença, verificamos concordância de $100 \%$ nos casos de agenesia renal, rim policísticos e obstrução uretral. Estas doenças cursam habitualmente com oligoidrâmnio, sendo seu estudo ecográfico beneficiado também pela amnioinfusão. Os equívocos diagnósticos se concentraram nas doenças obstrutivas (concordância de 85,3\%), em que verificamos que houve erro na definição do nível de obstrução e confusão diagnóstica com rim multicístico. A literatura aponta este último como a principal fonte de erro diagnóstico, pois a imagem 
ecográfica de megaureter e hidronefrose, quando mais avançados, pode simular os cistos de parênquima renal vistos no rim multicístico. São duas doenças que exigem condutas diferentes, uma vez que a primeira permite tratamento intrauterino, caso a função renal esteja preservada, ao passo que para a segunda, o rim acometido cursa invariavelmente com perda da função. O estudo ecográfico seriado nos parece um recurso útil para esclarecer o diagnóstico diferencial.

Outros estudos de concordância diagnóstica pré e pós-natal, seja na definição morfológica da nefrouropatia fetal, bem como na avaliação funcional do rim, são de grande utilidade para permitir abordagem pré-natal adequada e melhorar a evolução perinatal e a longo prazo destes individuos.

O diagnóstico morfológico das nefrouropatias fetais, por meio da sistematização do estudo ecográfico, apresenta excelente concordância com o diagnóstico pós-natal, com índice de kappa de $95 \%$. Ao definir no pré-natal a natureza da nefrouropatia, é possivel adotar conduta obstétrica no sentido de preservar a função renal pósnatal ou minimizar os danos a ela.

\section{SUMMARY}

Purpose: to evaluate the accuracy of prenatal ultrasound in the diagnosis of nephrouropathies.

Methods: the authors followed-up 127 pregnancies referred to the Fetal Medicine Center of UFMG with suspicion of these anomalies. Fetal biometry, growth, vitality, and associated malformations were evaluated. Finally, a detailed description of the renal system was made to define the prenatal morphologic diagnosis of the malformations to be compared with the postnatal diagnosis.

Results: based on the kappa index (statistical method that measures the concordance between different measurements, methods or measurement instruments: below 0.40, poor agreement; between 0.40 and 0.75 , good agreement; above 0.75 , excellent ageement), the authors found an excellent concordance (kappa index 0.95). Among the 127 cases, there were only 9 misdiagnoses, all of them of obstructive uropathies: 6 cases showed different obstruction levels after delivery and in three cases there were confounding diagnosis with multicystic kidney.

Conclusions: the detailed ultrasonographic description of the renal system is a good method for prenatal diagnosis of the fetal nephropathies, allowing some options to modify the outcome of these fetuses, like to send them to specialized centers, to anticipate delivery and even to apply intrauterine therapy, in order to preserve the renal function. Serial echography and amnioinfusion can be used to improve the precision of prenatal diagnosis.

KEY WORDS: Fetal malformation. Ultrasound. Prenatal diagnosis. Fetal nephrouropathies.

\section{Referências}

1. Carrera JM. Diagnóstico prénatal. Barcelona: Salvat; 1987. p.3-9.

2. Patten RM, Mack LA, Wang KY, Cyr DR. The fetal genitourinary tract. Radiol Clin North Am 1990; 28:115-30.

3. Nicolaides $\mathrm{KH}$, Cheng $\mathrm{HH}$, Abbas A, Snijders RJ, Gosden C. Fetal renal defects: associated malformation and chromosomal defects. Fetal Diagn Ther 1992; 7:1-11.

4. Sullivan KM, Adzick NS. Fetal surgery. Clin Obstet Gynecol 1994; 37:355-71.

5. Thomas DF, Gordon AC. Management of prenatally diagnosed uropathies. Arch Dis Child 1989; 64:58-63.

6. Diniz JSS. Insuficiência renal crônica na criança: relato de uma experiência no HC/UFMG. Rev Med Minas Gerais 1993; 3:127-33.

7. Garcia C, Goldani J, Garcia V. Paediatric dialysis and renal transplantation in the state of Rio Grande do Sul, Brasil. Pediatr Nephrol 1992; 6:74-7.

8. Oliveira EA. Estudo prospectivo das anomalias do trato urinário diagnosticadas no feto pelo ultrasom [dissertação]. Belo Horizonte: Universidade Federal de Minas Gerais; 1992.

9. Malone PS. Antenatal diagnosis of renal tract anomalies: has it increased the sum of human happiness? J R Soc Med 1996; 89:155-8.

10.Cabral ACV, Leite HV, Aguiar RALP, Oliveira EA. Tratamento da obstrução de uretra fetal. Relato de 2 casos. J Bras Ginecol 1992; 102:121-2.

11.Garrett WJ, Kossoff G, Osborn RA. The diagnosis of fetal hydronephrosis, megaureter and urethral obstruction by ultrasonic echography. Br J Obstet Gynaecol 1975; 82:115-20.

12.Paduano L, Giglio L, Bembi B, Peratoner L, D' Ottavio G, Benussi G. Clinical outcome of fetal uropathy. I. Predictive value of prenatal echography positive for obstructive uropathy. J Urol 1991; 146:1094-6.

13.Tam JC, Hodson EM, Chong KK, et al. Postnatal diagnosis and outcome of urinary tract abnormalities detected by antenatal ultrasound. Med J Aust 1994; 160:633-7. 
14.Vilits P, Ring E, Petritschi PH, Rauchenwald M, Colombo T, Hubmer G. Prenatal diagnosis of abnormalities of urinary tract. Wien Klin Wochenschr 1995; 107:736-8.

15.Podevin G, Mandelbrot L, Vuilard E, Oury JF, Aigrain Y. Outcome of urological abnormalities prenatally diagnosed by ultrasound. Fetal Diagn Ther 1996; 11:181-90.

16. Harrison MR, Ross NA, Noall RA, de Lorimier AA. Correction of congenital hydronephrosis in utero I. The model: fetal urethral obstruction produces hydronephrosis and pulmonar hypoplasia in fetal lambs. J Pediatr Surg 1983; 18:247-56.

17.Sholder AJ, Maizels M, Depp R, et al. Caution in antenatal intervention. J Urol 1988; 139:1026-9.

18. Chierici R, Riccipetitoni G, Tamisari L, et al. Conservative management of urinary abnormalities detected in utero. Fetal Ther 1989; 4:43-8.
19.Livera LN, Brookfield DS, Egginton JA, Hawnaur JM. Antenatal ultrasonography to detect fetal renal abnormalities: a prospective screening programme. BMJ 1989; 298:1421-3.

20.Rosendahl H. Ultrasound screening for fetal urinary tract malformations: a prospective study in general population. Eur J Obstet Gynecol Reprod Biol 1990; 36:27-33.

21.Korantzis A, Cardamakis E, Apostolidis C. Prenatal diagnosis of fetal urinary pathology with ultrasound. Eur J Obstet Gynecol Reprod Biol 1993; 52:169-74.

22.Lopes APBM. Tratamento das uropatias obstrutivas fetais utilizando cateter desenvolvido no Centro de Medicina Fetal do Hospital das Clínicas da UFMG [dissertação]. Belo Horizonte: Universidade Federal de Minas Gerais; 1997.

\section{Informações: Sociedade de Ginecologia e Obstetrícia do Rio Grande do Norte}

